\title{
Analyzing Improvements for a Mine Maintenance System of Connected Equipment and Machines
}

\author{
The Value and Benefits of Data Sharing
}

\author{
Joakim Fröberg, Stig Larsson \\ SICS Swedish ICT AB \\ Sweden \\ [joakim.froberg][stig.larsson]@sics.se
}

\author{
Ulf Marklund \\ System Technology \\ Boliden Mines \\ Boliden, Sweden \\ ulf.n.marklund@boliden.com
}

\begin{abstract}
A modern mine involves increasingly smart and connected products that are integrated in a mine automation system. Integration enable many possible applications that could substantially aid in achieving the goals of increased safety and productivity of the mine operation including the machine maintenance process. What data will be shared by the involved organizations and products, heavily affects how successful improvements of operation can be accommodated.
\end{abstract}

We have devised a method to map out and evaluate envisioned new collaborative functions for a complex System-ofsystems such as the mine maintenance operation. The proposed method map user stories for the involved stakeholders and estimate the value of fulfillment based on different candidates of data sharing architectures. The method is explained and exemplified by a realistic example based the real case.

There seems to be a need for a method such as the presented one just to map out what new applications are really feasible. By estimating value in terms of stakeholder benefits and identifying possible showstoppers in terms of protected data, the method seems to help reveal what improvements in the mine operation is in fact possible. Deciding on a data-sharing architecture for a collaborative mine seems to provide useful design prerequisites to a developing organization improving their smart connected products.

Keywords-System architecture, architecture analysis, mine maintenance, system-of-systems, data sharing architecture

\section{INTRODUCTION}

The automation of operations is a top technological driver for today's mining companies [1]. Modern products, including mine equipment and mining machines are increasingly becoming smart connected products [2] and are integrated into collaborative mine automation systems. For a mine operator, the goal of mine automation is to increase the safety, productivity, and quality of the mine operation. This includes goals of more efficient and effective procedures such as maintenance and monitoring of diagnostic and production status. By combining signals from connected systems, there are many possible applications that could substantially aid in achieving the goals of an enhanced mine operation [3]. For instance, combining diagnostic data from a Load-Haul-Dump machine, LHD, with production, and maintenance planning systems could enable a much more efficient service planning procedure. Or, combining position data from several systems could yield a much better precision and thereby enable new safety mechanisms that preempts and avoids collisions with people or machines.

Mining machines and other equipment, as well as mine automation systems come from many developing organizations, and are themselves commercial products that are integrated into the mine automation system. A modern mine is thus an example of the trend towards the internet-of-things, IoT, more and more products are connected to the Internet and to a local networks [4]. In addition, vehicles or machines communicate to enable new features, so called machine-tomachine, or machine-to-infrastructure, M2X, communication. The mine system can be described as a System-of-systems, SoS, or a federated system, where several systems participate in collaborative goals, while also performing specific tasks.

Many methods for systems engineering and system architecture analysis are formed on the basis that we analyze the stakeholder needs and quality attributes, and thereby come up with an architecture that best support these needs. In a SoS, each system is a product with its own life cycle and, in this case, also a business model and business value.

For each organization, there are constraints related to the effectiveness of its own products. Both from a design perspective and a business perspective. Our study indicate that there is a large potential value in sharing data, both for the mine operator and for the product vendors, whether it is a machine OEM or an equipment vendor. The problem initially lies in the ability to analyze the overall potential for new value creating features, and to relate that to what signals and data sharing that is beneficial - both for a system supplier, integrators and for a mine operations organization. Without this knowledge, an organization may attempt secrecy of outgoing data, while trying to improve own product performance by incoming data - this would be a suboptimal design for all, we believe.

We have devised a method to map out any envisioned new collaborative functions for a complex SoS such as the mine 
operation. We relate the collaborative goals to the needs of shared signals, and thereby indicate a business value of shared data. Our method visualize, and categorize data sharing needs. It aids in analyzing the value of data in the specific usage context - in our case study, the mine operation. Our method can support analysis of a complex SoS system and visualize business value to each involved organization. This would aid in understanding what data is valuable to share, in an operation such as mine maintenance.

The case data and analysis is based on interviews and discussions we have had within the project Wroom (Wireless and remote operation of mobile machines) a collaborative project with industry and research partners. The project is part of the Process Industrial IT and Automation (PiiA) strategic innovation program funded by the Swedish research funding organization Vinnova.

The contribution of this paper is the proposed method for architecture analysis for a cooperative architecture of connected data sharing systems. The proposed method is explained by using the real world example of a mine maintenance context

In section 2, we outline the method. In section 3, we show an example based on our study of the mine case. Section 4 presents a discussion on using the method, while section 5 concludes the paper.

\section{A METHOD FOR ANALYSIS}

In this study, we are attempting to define an analysis method to design a collaborative architecture for the connected systems being part of a mine maintenance system.

We have previously tailored a workable method for architecture analysis [5][6] based mostly on the MFESA [6] framework. This work led to a nine step method describing the procedure of how to elicit architecture drivers, identify architectural candidates and evaluate appropriateness.

In this study we are faced with a system that involve several organizations that have a collaborative goal, but also their own strategies for data sharing and product development. The mine operation involves much technical equipment such as loader type machines, many other types of machines, many other types of equipment, a process control system, a mine operation control system, a maintenance operation system. All of these are connected products.

The mine operation process involves a number of stakeholders that work to achieve a collaborative goal - the production of materials with a high safety and predictably high productivity. We have studied the stakeholder needs for improvements of the maintenance operation of the mine and elaborated on the technical architecture that is needed to satisfy those needs.

An engineering method of architecture analysis aims at analyzing the crucial drivers, and success factors of an intended system [8][9]. The analysis should provide basis for evaluating and selecting the best architecture for the job. The point is that the system is intended for, and evaluated in the light of, an overall goodness of the system in fulfilling the needs. For a system of connected products, each of whose organization has their own goals, the goodness of the system is heterogeneous. Improving the overall mine procedure is a target for all the cooperating organizations, but also to care for the own product in terms of servicing, soft services, and data intellectual property.

For this purpose, we have tailored a method that should map out the collaborative data sharing architecture of a system of systems that involve several organizations with products and data sharing strategies. The method is tailored by using our previous nine step method [6] and specialize it towards a collaborative architecture. We have applied it to a production system for mine involving loader machines, and we have specifically aimed it at analyzing the maintenance process.

In order to analyze the architecture needs of each organization, there is a need to find out how the organizations will and will not collaborate in terms of data sharing. Most products are connected and thereby much new opportunities arise. For instance, the mine production can be optimized and made more efficient by combining data from the diagnostic data in the machine, with mine operation scheduling, and maintenance system, so as to minimize downtime and unnecessary stops. So, for each organization there is a need to clarify a general concept of what data can and will be shared. Only after that, each organization can do a regular architecture analysis and evaluate which architecture is best for the purpose. Principal collaborative principles must be established. Basically there will be questions of - Will our product have access to a particular data, and with what precision, and update frequency? Also issues of data format will arise and wireless reliability etc.

The idea of the method is to map out the communicated data signals between organizations, or at least meta-data on what type of data will be available.

\section{A STEPWISE METHOD TO ANALYZE A COLLABORATIVE DATA- SHARING ARCHITECTURE}

This is the stepwise method that we propose to perform the architecture analysis for the collaborative system of mine maintenance. The tailored method is simpler than a normal architecture analysis in the sense that it considers only the architectural issues around the data sharing between the collaborating organizations. All other quality attributes and concerns can be left out in this phase. The important focus is to define the communicated data, and identify any showstoppers. There are seven steps.

- Model Architectural drivers for the system in its complete life-cycle. Stakeholder interviews and workshops are used to produce user stories [10] to describe the system being used through the life cycle. Take note of stakeholders, success criteria, risks and opportunities. Each user story can be elaborated an include explanation of what defines a successful system use. Each life-cycle process is an aggregate of all its use-cases. 
- Reduce scope to what is considered feasible. Assign architectural risk and architectural opportunity based on team judgment to the usage model. Estimate severity and probability for each risk/opportunity and filter out too risky use-cases or user stories. In effect, this step reduces the scope and boundary of what the system is to do.

- Model the organizational data exchange involved in the overall operation. Identify the organizations and identify possible interfaces for data. Avoid distinguishing between different systems within each organization. (For instance, an intelligent transportation system may involve a weather service organization, several car OEMs, traffic infrastructure org and a telecom service provider.)

- Model data requirements. Start with the user story and identify which data is required to flow between organizations in order to fulfill the user story. There is likely many ways to accommodate the end result, but try to outline the ones that differ substantially. Work through the set of user stories for a given category, e.g., the maintenance operation of the collaborating system. Compile the data requirements for all those user stories in one diagram. This diagram represents one principal solution, an architecture candidate, of data sharing that solves the stipulated user stories.

- Validate the feasibility of each organization to generate and signal the defined data. Validate the precision, data-rate, and reliability of data against the data requirements.

- Compare and evaluate candidates. Estimate how well a certain candidate fulfills a user story as expressed by one stakeholder. Estimate a value, e.g., what is the value of completing a certain task faster or more accurately. For each organization, sum up the values. This will indicate how well the architecture will support the users from that organization.

- Analysis of value. Identify possible problems. Validate feasibility for the use of the involved data. Some data content may be sensitive or proprietary for strategic reasons. On the other hand, if the value is high enough, partners can trade, or subscribe to data. At the least this analysis should help identify potential show-stoppers, but also indicate possible solutions in terms of data services.

Now, we have a definition of the data sharing; what data is produced and consumed in what organization. We have a definition of the different architectures that can support all the user-stories. And we have a notion of the value of the architectures indicated by each organization. The simplified architecture analysis should have made sure that the data sharing architecture do support each organizations need on the system. The main idea with getting to this point of agreement around the data sharing is that each organization is now aware of the prerequisites for how their systems should work to support the collaborative goals. A normal architecture analysis can be performed on an individual system or product without the uncertainty of what is and what is not shared.

\section{USING THE METHOD - AN EXAMPLE}

We present the use of the method by an example. The example is simplified in order to be presentable, but based on the case. The exact data and architecture candidates are not corresponding with those in the real case.

\section{A. Model Architectural drivers for the system in its complete life-cycle.}

We modeled the envisioned system by using user stories. There are different but similar formats on user stories, and we chose a common format as proposed by Mike Cohn [10].

\footnotetext{
"As a <type of user>, I want <some goal> so that <some reason $>$ "
}

The idea is to capture the stakeholder that is interested in the result, the wanted outcome itself, and the reason for it. In order to assure that the story is understood, we add a description of what is the acceptance criteria for the story to have a successful outcome. We documented the user stories and acceptance criteria as expressed by the interviewees. We use this one story as an example throughout this example.

User story: "As a service planner I want to know when a machine need to be serviced and what type of service, in order to prepare the resources, time, spare parts of the service stop."

\section{Acceptance criteria:}

- The service planner get the information on service needs on the machine (level of remaining expendables, the level of wear of exchangeables)

- The service planner is made aware if the machine status is unavailable or faulty.

- The supply planner gets information on what spare parts to order and when.

\section{B. Reduce scope to what is considered feasible.}

There are user stories that, when elaborated, are shown to be unfeasible in some way. In the mine maintenance context, there could be user stories that wish for machines that selfschedule their own maintenance or re-plan production automatically. If this is considered too risky in terms of development effort or productivity, those user stories can be left out or postponed, effectively setting the scope for what the system is to do and also set a boundary on what data consequently must be shared.

\section{Model the organizational data exchange involved in the overall operation.}

Without elaborating on which technical systems that are involved, we outline which organizations are involved in the exchange of data. Considering only the one user story, we can identify what data is required for this story to be supported.

By thinking of what data is needed to successfully execute the story, we identify one way of routing the signals among data owners and consumers. For example, the machine status needs to be signaled from a machine and then handled by a 
control system and connected to a mine system. The mine system can send alarms and transactional data to the machine maintenance system where the service planner can make an informed decision on when and how the service is to be done. In order to get the best estimate for upcoming service needs, the operational plan can be combined with machine status and analyzed by the OEM service organization.

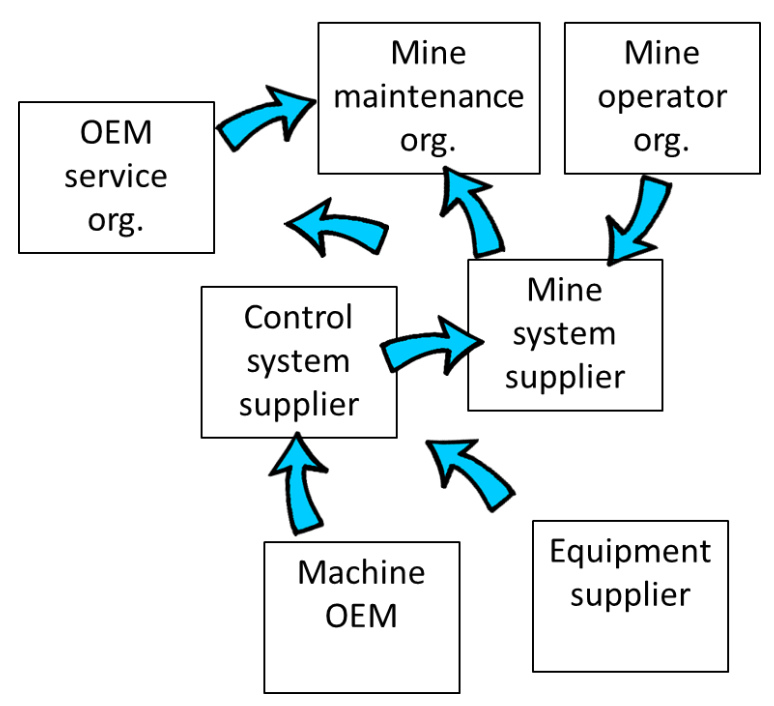

Fig. 1. One graph to outline the data routing between organizations.

This is one principal way that the data sharing could be routed. There are more ways and we take note of alternatives by documenting one graph like the one in Fig 1 for each proposal. Then we proceed by working through all the user stories that are relevant for the maintenance operation. Now we have a set of data needs represented by arrows and hopefully a few different principal ways of solving the data sharing.

\section{Model data requirements.}

For each arrow in the figures, we take note of the specifics. What data is needed? We define precision, format, and update rate. We add the data requirements specifications in an organization square matrix. A set of data signals are defined and given an identifier and inserted in the matrix corresponding to which organizational interface is used. Below in figure 2 is a few data carriers shown in the diagram.

For instance, there could be a signal of hydraulics oil quality of a machine. This could be sent and possibly refined in the process control system, and then to be analyzed in combination with work order data at the mine operator organization.

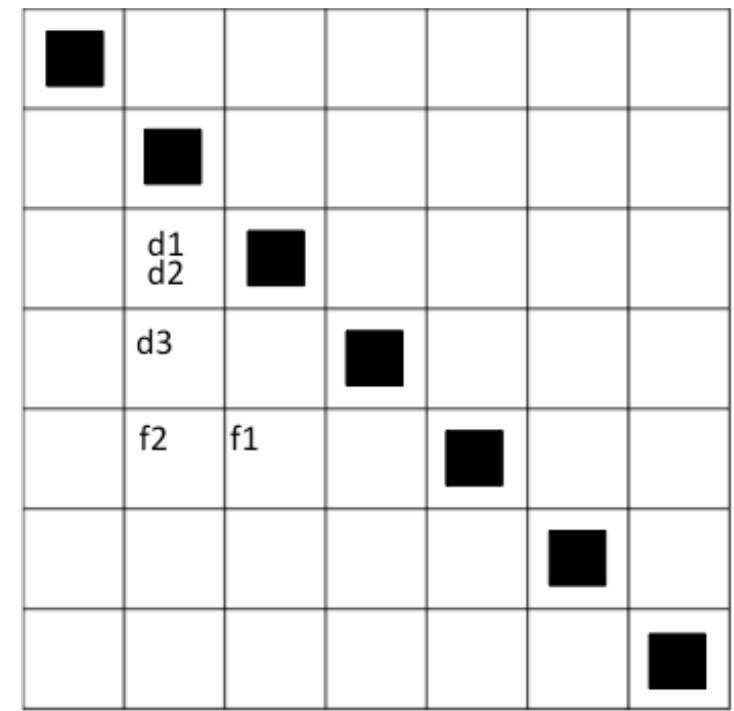

Fig. 2. Data sharing organizational matrix.

The end result could be a best estimate of how long time the machine has before the service stop has to be planned. Those data carriers would be added in the diagram showing where the data is produced and consumed, and thus the diagram represent one possible data sharing architecture. We complete a matrix diagram each for the valid proposals of data sharing architectures.

\section{E. Validate the feasibility of each organization to generate and signal the defined data.}

We go through all the signals and make sure that they fulfill the needs of the user stories. Also that they are feasible to produce by the data owners and that they are eligible for sharing, trading or subscribing to.

\section{F. Compare and evaluate candidates.}

For this example with only one user story, we exemplify two ways of supporting the service planner. Either just combine machine status data with mine operation plan, and thereby come up with an estimate for when the service stop should occur, or perform the added signaling to involve also the OEM to do statistical analysis. In order to compare two options such as these, it is possible to compile all the benefits perceived by each stakeholder. How much better would the service plan be if we go for the more complicated data sharing? It seems wise to categorize all benefits per organization.

\section{G. Analysis of value.}

In order to be able to choose between candidates, it could be a good idea to try and assign a value to a successful execution of a user story. For instance, how much service costs could be avoided by getting a better service plan? How many unplanned stops could be avoided per year? Those measures can sometimes be estimated and give a good notion of how much value can be expected for a certain candidate architecture. Each architecture can be denoted with the sum of all the values of all improvements as estimated by the stakeholder in each organization. 


\section{DISCUSSION}

The trend towards the Iot with connected systems in mines and elsewhere seems to enable a large number of new applications. There seems to be a need for a method such as the presented one just to map out what new applications are really feasible. By estimating value in terms of stakeholder benefits and identifying possible showstoppers in terms of protected data, the method seems to help reveal what improvements in the mine operation is in fact possible.

Also, for each organization that is involved, there seems to be a need to define the premise for collaboration, in order to be able to design and optimize the own products. Once the data sharing architecture has been decided it is possible to perform architecture analysis on each system, an activity that would be challenging without an agreement on how data should be shared.

It seems important to note that a normal architecture analysis performed on the complete system-of-systems would be difficult because of the heterogeneous goals of the organizations. Instead, we attempt an adapted analysis method based on the assumption that data sharing could lead to benefits to all stakeholders although they strive towards different goals. The goals are not contradictory, so it seems a reasonable assumption.

It is crucial to let the organizations identify what improvements and what values are possible to achieve for their own stakeholders. This can be indicated in our example by considering the two viewpoints of the mine operator and the machine OEM respectively. The mine operator values the availability of production at all times, but do not have the statistical data to predict unplanned stops. The OEM has the statistics based on many machines, but do not have the full context of how the machine is used in the specific mine. These two viewpoints value user stories differently, and thus value different pieces of shared data. Rightly planned, the data sharing of these two organizations would enable improvements in the life cycle of both organization.

\section{CONCLUSION}

In this study we are faced with a mine automation system that involve connected products of several organizations that have a collaborative goal, but also their own strategies for data sharing and product development.

We have tailored a method to map out the collaborative data sharing architecture of a system of systems that involve several organizations involved in the mine automation system.
We have applied it to the mine operation and specifically the maintenance process of LHD machines.

The method utilize user stories to map out stakeholder needs of the different organizations. Inter-organization data sharing is mapped out by diagrams, each one representing the data content and data routing of one candidate architecture. Each candidate can then be assigned with a value by estimating all improvement potential for all the stakeholders.

There seems to be a need for such a method in order to map out what new applications are really feasible in an IoT setting for a modern mine. It identifies possible showstoppers in terms of protected data and the method seems to help reveal what improvements in the mine operation is in fact possible. Deciding on a data-sharing architecture for a collaborative mine seems to provide useful design prerequisites to a developing organization improving their smart connected products.

\section{REFERENCES}

[1] Lee, J., Prowse, K., "Mining \& Metals + Internet of Things: Industry opportunities and innovation", MaRS Discovery District market report, http://www.marsdd.com/news-and-insights/mining-industry-iottechnology/

[2] Porter, M.,Heppelmann, J., "How Smart, Connected Products Are Transforming Competition", Harvard Business Review, Nov 2014.

[3] McBeath, B., "How the Internet-of-Things is Transforming Mining", http://www.clresearch.com/research/detail.cfm?guid=A5E6FEAF-304879ED-99C9-EA62EBCCD605\#ftn1Return

[4] Prowse, K., "The Internet of Things in Mining: A Billion-Dollar Toy Store", Techvibes, Dec 2014, http://www.techvibes.com/blog/miningthings-internet-2014-12-09

[5] Fröberg, J., Larsson, S., and Nordlander, P., "A Method for Analyzing Architectural Drivers When Engineering a System Architecture." IEEE International Systems Conference, Orlando, Florida, 2013.

[6] Froberg, J.; Larsson, S.; Dersten, S.; Nordlander, P.-A., "Defining a method for identifying architectural candidates as part of engineering a system architecture," IEEE Systems Conference (SysCon), 2014

[7] Firesmith, D.G., Capell, P., Hammons, C.B., Latimer, D.W., and Merendino, T.: "The Method Framework for Engineering System Architectures," Taylor \& Francis, 2008.

[8] P. Clements, R. Kazman, M. Klein, "Evaluating Software Architectures - Methods and Case Studies," SEI Series in Software Engineering, Addison-Wesley, 2002.

[9] Barbacci, Mario; Ellison, Robert; Lattanze, Anthony; Stafford, Judith; Weinstock, Charles; Wood, William. "Quality Attribute Workshops (Qaws), Third Edition, Technical Report Cmu/Sei-2003-Tr-016." 2003.

[10] Cohn, M., "User Stories Applied: For Agile Software Development", Addison Wesley, 2004. 